\title{
Stress estimation in reservoirs using an integrated inverse method
}

\author{
Antoine Mazuyer ${ }^{1}$, Paul Cupillard ${ }^{1}$, Richard Giot $^{2}$, Marianne Conin $^{1}$, Yves Leroy ${ }^{3}$, and Pierre Thore ${ }^{3}$ \\ ${ }^{1}$ GeoRessources (UMR 7359, Université de Lorraine / CNRS / CREGU), 54505 Vandouvre-lès-Nancy, France \\ ${ }^{2}$ IC2MP (UMR 7285, Université de Poitiers / CNRS), 86073 Poitiers, France \\ ${ }^{3}$ Total S.A, CSTJF, Avenue Larribau, 64000 Pau, France
}

\begin{abstract}
Estimating the stress in reservoirs and their surroundings prior to the production is a key issue for reservoir management planning. In this study, we propose an integrated inverse method to estimate such initial stress state. The 3D stress state is constructed with the displacement-based finite element method assuming linear isotropic elasticity and small perturbations in the current geometry of the geological structures. The Neumann boundary conditions are defined as piecewise linear functions of depth. The discontinuous functions are determined with the CMA-ES (Covariance Matrix Adaptation Evolution Strategy) optimization algorithm to fit wellbore stress data deduced from leak-off tests and breakouts. The disregard of the geological history and the simplified rheological assumptions mean that only the stress field, statically admissible and matching the wellbore data should be exploited. The spatial domain of validity of this statement is assessed by comparing the stress estimations for a synthetic folded structure of finite amplitude with a history constructed assuming a viscous response.
\end{abstract}

\section{Keywords}

Inverse Problem

3D stress field

Integrated libraries

\section{INTRODUCTION}

In-situ stress state knowledge is particularly essential in civil engineering (tunnelling or excavations), mining (setting mine plans) and in the petroleum industry. During oil and gas field exploitation for instance, the depletion disturbs the mechanical equilibrium, yielding a stress change in the reservoir and the overlying geological layers (the overburden) [Addis, 1997]. This could have unexpected consequences such as borehole instabilities [Zoback et al., 2003] or oil and gas leaks or migration [Wiprut and Zoback, 2002]. Such risk can be identified with yield criteria such as the Mohr-Coulomb criterion which depends on the stress state. The goal of this paper is to present an integrated method to compute the virgin stress in reservoirs and surrounding rocks. The method relies on an inverse approach. It is called "integrated" because it is not specific to a given case study and it can handle adjustable numerical methods and software. Another significant advantage of the method is the proper account of complex 3D geometries.

In-situ methods such as relaxation methods [Amadei and Stephansson, 1997], leak-off tests [Clark, 1949] or borehole breakout analysis using wellbore images [Zoback, 2007] give a one dimensional stress estimation. They partially estimate the 3D stress tensor, but they all suffer from the sparsity of observations and the scarcity of stress measurements. Moreover, interpolating the stress field between local measurements is extremely challenging and questionable because of the material and mechanical heterogeneities such as faults or folds that highly disturb it [Faulkner et al., 2006, Zoback et al., 1987]. Therefore, in-situ methods are not sufficient to fully estimate the stress field in 3D.

An approach to estimate the stress field in a whole 3D domain is to numerically solve Partial Differential Equations describing the geological history and the rheology of the medium studied. Such an approach requires the knowledge of all the tectonic events from the sediment deposits to the current geological layers geometry. It also requires the mechanical behaviours of the materials to be known during all the history. At this time-scale, these behaviours involve time-dependent non-linear constitutive models such as plastic, visco-elastic or visco-elasto-plastic laws. For instance, Hassani et al. [1997] developed a visco-elastic model to study the plate deformation and stress in subduction processes, and Gerya and Yuen [2007] applied a visco-elasto-plastic rheology to subduction zones, magmatic dikes, etc. The implementation of such models in computational codes is very tedious and involves a high computational cost. Furthermore, the precise geological history and mechanical behaviours are not known. As a result, reproducing the whole geological history is not a good solution for our purpose, namely developing a case-independent and numerically flexible method.

The key point of this study is to consider the current geometry of the geological structures and to not take into account the geological history by constraining the stress estimation to the wellbore data. A similar idea has been investigated by Madyarov and Savitski [2010]. These authors limit their work to a simple 2D test and use a classical gradient-based algorithm to minimize the misfit between the data and the computed stress by changing the Neumann conditions (i.e. the prescribed forces on the model boundaries). Maerten et al. [2016] have also studied the estimation of the "far field tectonic stress" by constraining the stress using the mechanical type, the orientation and the location of fractures. They use the boundary element method to compute the stress, which is suitable and fast for homogeneous elastic medium.

The method proposed in this paper is to use a simple linear elastic behaviour within a finite element method to take into account 3D heterogeneous medium. We set up an inverse problem where the model parameters (as defined by Taran- 
tola [2005]) are the Neumann conditions (Fig. 1a). These boundary conditions have no reason to match the real ones in place. Solving the linear elasticity equation within an inverse approach just guarantees to get an admissible stress field in the whole domain (Fig. 1b). The CMA-ES algorithm is used to solve the optimization problem and update the Neumann conditions (Fig. 1c) as it shows good robustness to solve illconditioned problems [Rios and Sahinidis, 2013].

The contribution contents are organised as follows. Section 2 sets up the forward problem and justifies the methodology we choose to compute the 3D stress field. The misfit function between the computed stress and the observed data as well as the minimization of this function are presented in section 3 . In this section, we also detail the libraries that we developed and the combination of tools that we built to solve the forward problem on complex 3D geological models and to reduce the misfit functions using high performance algorithms. Finally, our method is tested in section 4 using a synthetic stress field generated using a large deformation simulation [von Tscharner and Schmalholz, 2015] as reference.

\section{THE FORWARD PROBLEM}

Table 1 defines all the symbols used in the paper.

Following Tarantola [2005], the forward problem links a set of model parameters $\boldsymbol{m}$ to a set of data $\boldsymbol{d}$ through an operator $\mathscr{F}$ such as:

$$
d=\mathscr{F}(m)
$$

In our case, the operator $\mathscr{F}$ expresses the mechanical system under study, which will be set in section 2.1. $m$ contains mechanical parameters and $\boldsymbol{d}$ contains the stress field values. The choice of $\mathscr{F}, \boldsymbol{m} \boldsymbol{d}$ and is driven by the hypothesis done on the mechanical behaviour. The result of the forward problem is the Cauchy stress field on a $3 \mathrm{D}$ domain $\Omega$ (e.g. a geological model).

\subsection{The mechanical system}

The operator $\mathscr{F}$ should not depend on the geological history. Stress computation is done on the current geometry of the geological layers. The assumption of the small displacement is made [Salençon, 2012]. As a consequence, the operator $\mathscr{F}$ can be defined as a boundary-value problem, which the associated strong form is:

$$
\left\{\begin{aligned}
\sigma_{i j, j}+f_{i} & =0 & & \text { in } \Omega \\
\sigma_{i j} & =C_{i j k l} \epsilon_{l k} & & \text { in } \Omega \\
u_{i} & =g_{i} & & \text { on } \Gamma_{g} \\
\sigma_{i j} n_{j} & =h_{i} & & \text { on } \Gamma_{h}
\end{aligned}\right.
$$

where $f$ is the external force, $\boldsymbol{C}$ is the elasticity tensor, $\boldsymbol{u}$ is the displacement vector and $\epsilon$ is the strain tensor. The domain $\Omega$ is bounded by $\Gamma$ as $\Gamma=\Gamma_{g} \cup \Gamma_{h}$ and $\Gamma_{g} \cap \Gamma_{h}=\emptyset . g$ is a prescribed displacement on $\Gamma_{g}$ (i.e. Dirichlet condition), $\boldsymbol{h}$ a prescribed force on $\Gamma_{h}$ (i.e. Neumann condition) and $\boldsymbol{n}$ is the normal to $\Gamma_{h}$. The weak form is set from 2 and is solved using a displacement based finite element method following Zienkiewicz and Taylor [2000]. Solving the weak form guarantees the stress solution

\begin{tabular}{|c|c|}
\hline Symbol & Definition \\
\hline \multicolumn{2}{|r|}{ Rock properties } \\
\hline$E$ & Young's modulus \\
\hline$v$ & Poisson's ratio \\
\hline$C$ & Elasticity tensor \\
\hline$\rho$ & Density \\
\hline$\eta_{\text {eff }}$ & Effective viscosity \\
\hline M & $\begin{array}{l}\text { Subscript that identifies the matrix of the syn- } \\
\text { thetic model }\end{array}$ \\
\hline$L$ & $\begin{array}{l}\text { Subscript that identifies the layer of the synthetic } \\
\text { model }\end{array}$ \\
\hline \multicolumn{2}{|r|}{ Mechanical quantities } \\
\hline$u$ & Displacement vector \\
\hline$\epsilon$ & Strain tensor \\
\hline$\sigma$ & Cauchy stress tensor \\
\hline$f$ & External forces \\
\hline$g$ & $\begin{array}{l}\text { Prescribed displacement (i.e. Dirichlet condi- } \\
\text { tion) }\end{array}$ \\
\hline$h$ & Prescribed force (i.e. Neumann condition) \\
\hline$a_{i} z+b_{i}$ & $\begin{array}{l}\text { A part of a piecewise Neumann boundary condi- } \\
\text { tion }\end{array}$ \\
\hline \multicolumn{2}{|r|}{ Inverse problem related symbols } \\
\hline$d$ & Vector of data \\
\hline$d_{\mathrm{obs}}$ & Vector of observed/measured data \\
\hline m & Vector of model parameters \\
\hline $\boldsymbol{m}_{\text {prior }}$ & Vector of prior knowledge on model parameters \\
\hline $\mathscr{F}$ & Operator linking $\boldsymbol{m}$ to $d$ \\
\hline$S$ & Misfit function \\
\hline & Mathematical notation \\
\hline
\end{tabular}
to be statically admissible in the whole domain $\Omega$.
Repeated lower cases subscripts are summed up from 1 to 3 . Partial derivative with respect to $x_{i}$ are denoted by ${ }_{, i}$

Table 1 Symbol definitions.

\subsection{Inverted parameters}

The values of parameters such as elastic coefficients (e.g. Young's modulus $E$ and Poisson's ratio $v$ found in $C$ ), density $\rho$ (found in $f$ ) and the prescribed displacements and tractions have to be known for solving Eq. 2. Eq. 1 can be re-written as:

$$
\mathscr{F}(\{E, v, \rho, h, g\})=\{\sigma\}
$$

The inverse formulation aims at retrieving the model parameters honoring the observations through the operator $\mathscr{F}$. The seismic [Bosch et al., 2010] and wellbore data interpretation could lead to the elastic coefficients and density estimation. We can reasonably make the assumption that this information is sufficient to build the mechanical model and do not invert them. The only model parameters to invert are the Neumann conditions so Eq. 3 reduces to:

$$
\mathscr{F}(\{h\})=\{\sigma\}
$$

The reader has to keep in mind that the inverted Neumann conditions are not the real boundary forces in place. The ultimate goal of this study is to estimate the 3D stress field using the 


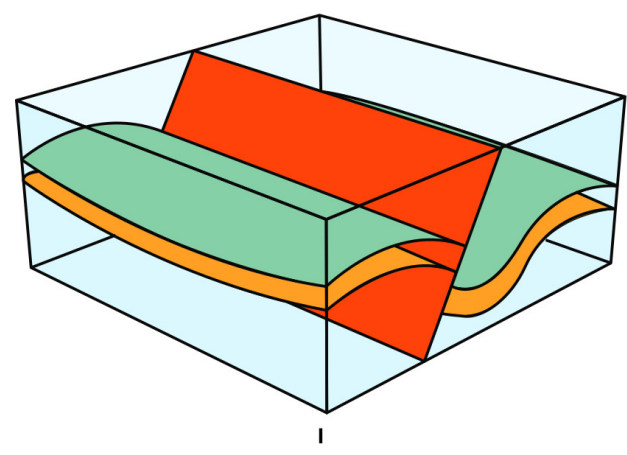

Meshing [Botella, 2016]

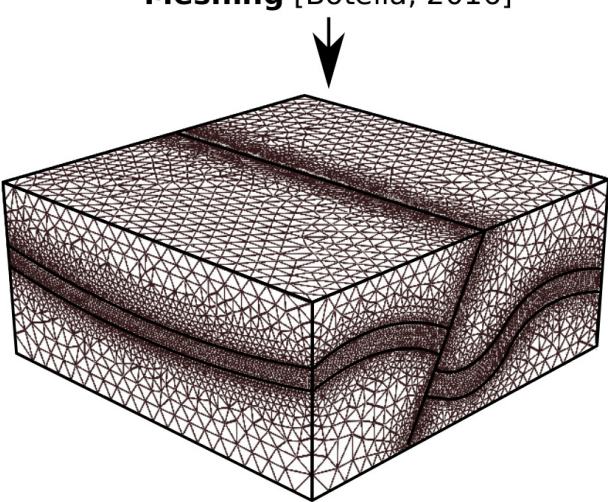

।

Property settings \&

Neumann conditions initialization

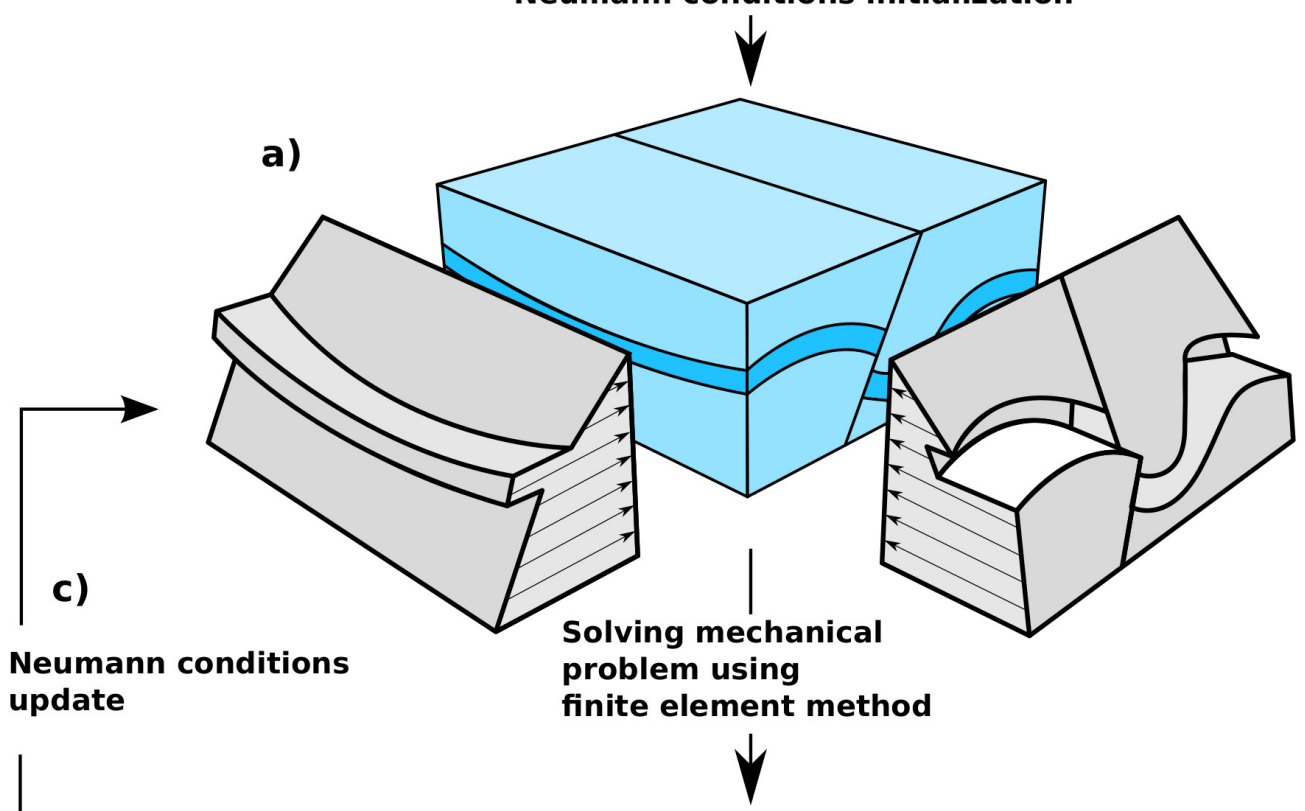

Gridding of the model with either a

tetrahedral or a hexahedral mesh

Density and elastic parameters are constant by layers

Neumann conditions are defined as piecewise linear functions

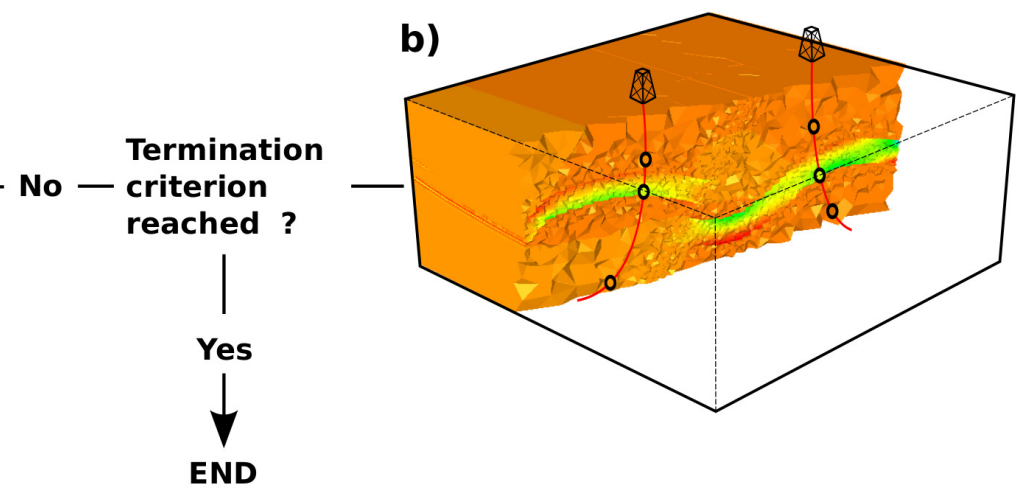

Section 2

Figure 1 The integrated method we propose to estimate stress state in rock mass constrained by observed data (taken along well-paths in this example). 
inverted Neumann conditions. Neumann conditions are here defined as piecewise continuous linear functions as Madyarov and Savitski [2010] proposed. These conditions are defined on two orthogonal faces of the model. The discontinuities are set where the horizons and the faults cross the boundaries. To simplify the mathematical expression of the applied forces, the model is supposed to be oriented in the direction of the principal stresses so the tangential components of the Neumann conditions vanish (even if the library used to solve the boundary-value problem can take into account forces which are non collinear to the face normals). A piece $i$ of the Neumann condition can be expressed as $: a_{i} z+b_{i}$ (Fig. 1a). Eventually, the inverse problem is summarized as the search for the couples $\left\{a_{i}, b_{i}\right\}$ for each piece of the Neumann conditions. Eq. 4 becomes:

$$
\mathscr{F}\left(\left\{a_{i}, b_{i}\right\}\right)=\{\sigma\}
$$

\section{ThE INVERSE PROBLEM}

\subsection{Available data}

In-situ stress field can be locally estimated using several methods that lead to a partial knowledge of the stress tensor. Relaxation methods are often used in civil engineering [Amadei and Stephansson, 1997]. The principle is to isolate a rock sample and to measure its displacement or deformation response. Then, knowing the mechanical behaviour, the stress tensor is deduced. Hydraulic fracturing [Clark, 1949] is used in civil engineering and in the petroleum industry. It gives an estimation of the least principal stress magnitude and orientation [Hubbert and Willis, 1957]. Borehole imaging can be used to locate borehole breakouts and tensile failures. The analysis of these failures gives an estimation of the horizontal stress orientation and under certain hypotheses the estimation of the values of the principal stress tensor [Zoback, 2007]. All the information concerning the local stress field (orientation, magnitude and corresponding standard deviations) could be taken into account as long as they can be computed when solving the forward problem. Thus, the observable stress data are stored in a data vector $\boldsymbol{d}_{\text {obs }}$ (Eq. 1) whose dimension is fixed by the amount of data available.

\subsection{Misfit function}

The misfit function aims at measuring the difference between the observed data $\boldsymbol{d}_{\mathrm{obs}}$ and the computed data $\boldsymbol{d}$ as well as the difference between the prior information we have on the model and the current model. This is the function to be minimized during the inverse process. It can be written as:

$$
\begin{array}{l|l}
S: & \mathbb{R}^{n} \longmapsto \mathbb{R} \\
\boldsymbol{m} & \longmapsto S(\boldsymbol{m})=\frac{1}{2}(\underbrace{\left\|\mathscr{F}(\boldsymbol{m})-\boldsymbol{d}_{\text {obs }}\right\|^{2}}_{\text {data misfit }}+\underbrace{\left\|\boldsymbol{m}-\boldsymbol{m}_{\text {prior }}\right\|^{2}}_{\text {model misfit }})
\end{array}
$$

As mentioned in Sec 2.2, the purpose of the method is to retrieve an admissible stress field matching the data by modifying the boundary conditions. We do not expect to have meaningful boundary conditions. As a consequence, the "model" term of Eq. 6 can be dismissed. The misfit becomes:

$$
S: \mid \begin{array}{rll}
\mathbb{R}^{n} & \longmapsto \mathbb{R} \\
\boldsymbol{m} & \longmapsto S(\boldsymbol{m})=\left\|\mathscr{F}(\boldsymbol{m})-\boldsymbol{d}_{\mathrm{obs}}\right\|^{2}
\end{array}
$$

The misfit function has to be minimized using an optimization algorithm. There are basically two kinds of such algorithms : the ones based on the gradient evaluation of the misfit function, and the ones based on a random search in the model space [Tarantola, 2005]. The reader should be aware that each evaluation of the misfit function presented in Eq. 7 requires the solving of the Eq. 2. The gradient computation on such functions can be difficult to determine [e.g. Giot, 2004].

The random search methods are more suited to optimize such misfit functions. Many examples of use of such methods exist. We can cite the Simulated Annealing method [Kirkpatrick et al., 1983] [e.g. Deutsch and Cockerham, 1994, Beaty et al., 2002, Das, 2012], and the evolutionary algorithms [Bäck and Schwefel, 1993] such as the genetic algorithms [e.g. Gallagher and Sambridge, 1994, Das, 2016] or the algorithms based on the Evolution Strategies such as Differential Evolution algorithms [e.g. Das and Prasad, 2015] or CMA-ES (Covariance Matrix Adaptation Evolution Strategy) [Hansen and Ostermeier, 2001].

CMA-ES is a stochastic search algorithm. It has demonstrated strong performances for solving ill-conditioned problems compared to other derivative-free algorithms [Auger et al., 2009]. Moreover, unlike gradient based methods, CMA-ES can easily optimize functions with a large set of parameters. That is why we choose it to minimize the misfit function.

The different steps of the algorithm are detailed in A.

\subsection{Integrated tools to solve the forward and in- verse problems}

One objective of this work was to develop integrated libraries which can be easily run regardless the complexity of the studied case. The starting point of this workflow is to build a 3D sealed geological model using a geomodeler such as SKUAGocad [Paradigm, 2017]. We can build complex 3D geological models and easily modify the geometry of the geological structures such as faults and horizons. We have conducted internal works to develop gridding tools to build unstructured meshes on such 3D models, conformal to the faults and the horizons [Botella, 2016, Botella et al., 2016]. These tools allow a full control on the mesh size (e.g. local refinment around complex structures). The boundary-value problem defined in Eq. 2 is solved using a homemade library (RINGMecha) which proposes a finite element implementation based on the MFEM library [MFEM, 2017]. We use the implementation of the finite element solver available in MFEM so the forward problem can be run using MPI (Message Passing Interface) parallelization. MPI allows to distribute the computation on various computers to increase the finite element performances, which is a great advantage when dealing with large domains. The CMA-ES implementation is based on the C ANSI code [Hansen, 2016] and is encapsulated inside an optimization library (GNIR). To enforce the integration of these different libraries, they all depend on RINGMesh, a free programming library for developing mesh based geomodeling applications [Pellerin et al., 2017], so they share the same data structure and use its API (Application Programming Interface). As a consequence, the different libraries never require a disk access to exchange data. The following code snippet presents the way these libraries can be associated together to form an integrated software. 
Code 1 Code snippet to demonstrate how the different libraries and methods could interact to solve the inverse problem.

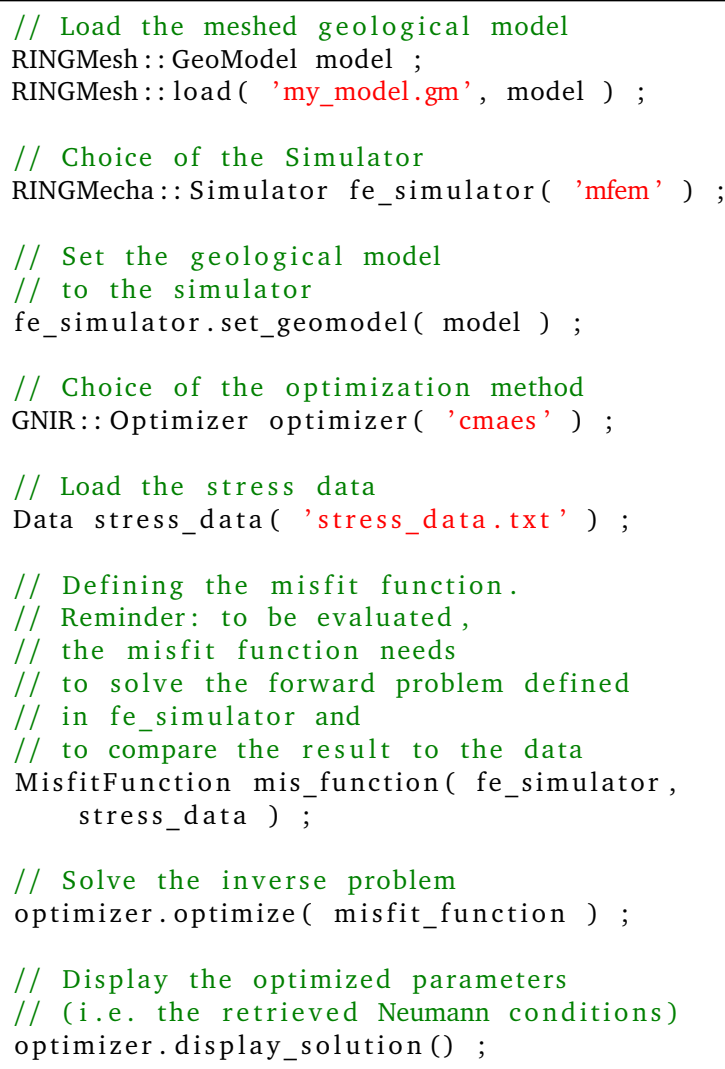

The strength of this workflow is that, even if here we use the finite element method to solve the boundary-value problem defined in Eq. 2 and choose the CMA-ES algorithm to optimize the misfit function, the libraries were developed with an abstraction allowing the developer to plug other optimization algorithms or other mesh based simulators to solve the inverse problem.

\section{TEST}

To test our method, a synthetic stress state is generated using a dynamic large-deformation simulation to generate a simple fold from a single thick flat layer embedded in a weaker matrix. The algorithm used to solve such problems is called PINK-3D and is presented in von Tscharner and Schmalholz [2015] (the code is available at the editor website as supporting information). The resulting stress is taken as reference stress and some values are extracted along virtual wells and are used as data for the inverse problem.

\subsection{Single layer folding using a large-strain algo- rithm}

In this section we use a 3D Lagrangian finite element algorithm to simulate a single layer folding embedded in a matrix.

We use this code on a 3D model with a strong thick layer embedded in a weaker matrix. The model is very similar to the one presented in section B3 of [von Tscharner and Schmalholz, 2015]. We use a layer thickness of $H=1$ and an initial total height of $H_{\text {model }}=21$. The stiffness difference is modeled with a ratio of 50 between the layer and the matrix $\left(\eta_{L} / \eta M=50\right.$, with $\eta_{L}$ and $\eta_{M}$ respectively the layer

\begin{tabular}{l|ccc}
\hline Surface & \multicolumn{3}{|c}{ Prescribed strain } \\
& $x$ & $y$ & $z$ \\
Top & none & none & $4 / 3$ \\
Bottom & none & none & 0 \\
West & 0 & none & none \\
East & -1 & none & none \\
North & none & $-1 / 3$ & none \\
South & none & 0 & none \\
\hline
\end{tabular}

Table 2 Boundary conditions applied for the single layer folding simulation. Orientation of the model is specified in Fig. 2.

and the matrix viscosity). The layer is initially perturbed following $A(x, y)=A_{0} \cos \left(k_{x}\right) \sin \left(k_{x}\right)$, where $k_{x}$ and $k_{y}$ are the wavenumbers in $\mathrm{x}$ and $\mathrm{y}$ direction. The reader may refer to von Tscharner and Schmalholz [2015] for more information on this perturbation and its evolution through time. Boundary conditions are summarized in Table 2.

All the parameters are dimensionless and are normalized using the layer thickness $H$ and the strain rate. The simulation is run with a time-step $\Delta t=0.01$ and it is stopped after 50 time-steps. Fig. 2 presents the initial configuration of the layer horizons and the resulting fold after the simulation.

Stress is computed iteratively during the simulation process using PINK-3D. Fig. 2 shows the resulting stress $\sigma_{x x}^{\text {ref }}$ in the $x$ direction on different slices of the model. The stress is mainly accumulated in the strong thick layer while it remains low in the matrix. The stress variation is also higher in the layer because of the fold which locally induces a compressive stress nearby the fold lower surface and an extensive stress nearby the fold upper surface. This computed stress field is taken as reference for testing the inversion. Nine virtual wells which are homogeneously distributed inside the model as shown in Fig. 2c, d, and e. $\sigma_{x x}$ and $\sigma_{y y}$ are extracted on five points per well. These extracted values are used as data for the inversion.

\subsection{Hypothesis}

The goal is now to test the method described in sections 2 and 3 and to compare the stress computed with the inverted Neumann conditions. The stiffness difference between the matrix and the layer is modeled with a ratio of 50 on the Young's moduli $\left(E_{L} / E_{M}=50\right.$ where $E_{L}$ and $E_{M}$ are the Young's moduli of the layer and the matrix). The Poisson's ratio is set to a constant value on the whole domain: $v=0.25$. The Neumann conditions are applied on the West and South boundaries. Because weight is not taken into account in PINK-3D, the $a_{i}$ coefficients of the model space defined in Eq. 5 vanish, so a model is reduced to $\boldsymbol{m}=\left\{b_{i}\right\}_{i \in \llbracket 0,5 \rrbracket}$. The $m(i)$ are detailed on Fig. 3. The first generation of $\left\{b_{i}\right\}$ for the CMA-ES algorithm is formed using an initial mean $\bar{b}=1$ and an initial standard deviation $\sigma_{b}=10$.

Comparing two simulation results can be very tedious. To limit these problems, the large-strain computation resulting mesh is reused as support for the finite element analysis during the inversion (Fig. 2b). To reduce side effects induced by the Neumann conditions, the South and the West boundaries are set far away from the zone of study (Fig. 3). These extensions will not be shown on the results for convenience. 


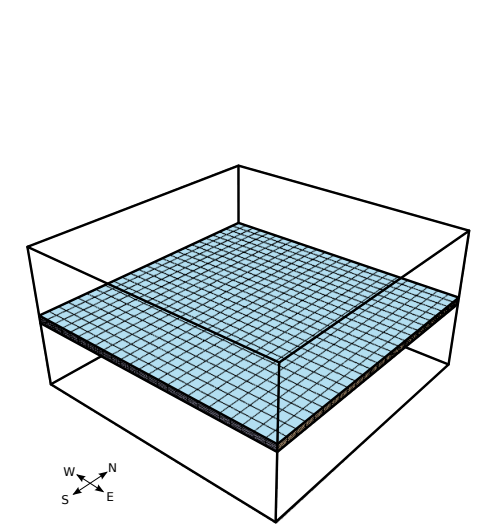

(a)

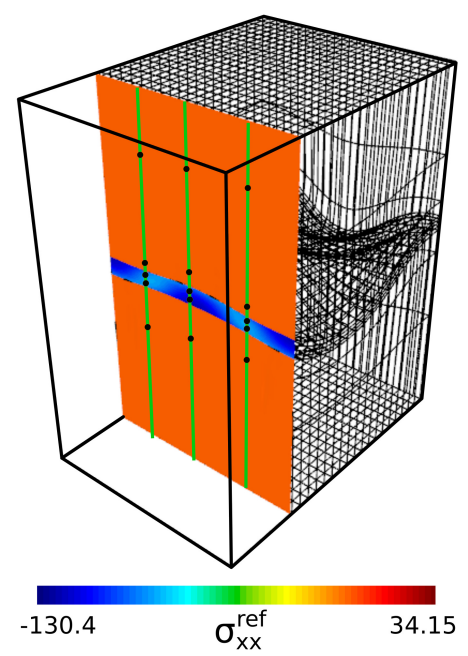

(c)

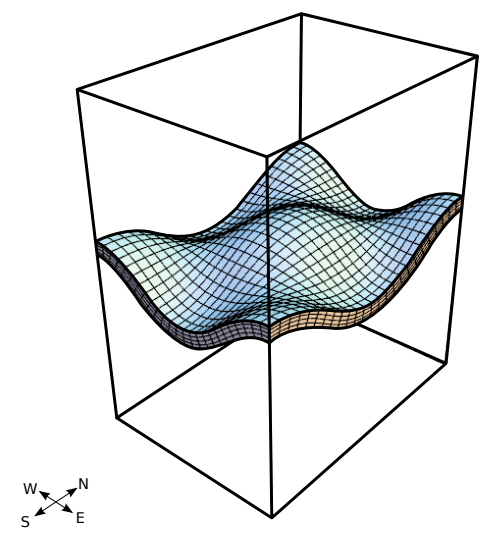

(b)

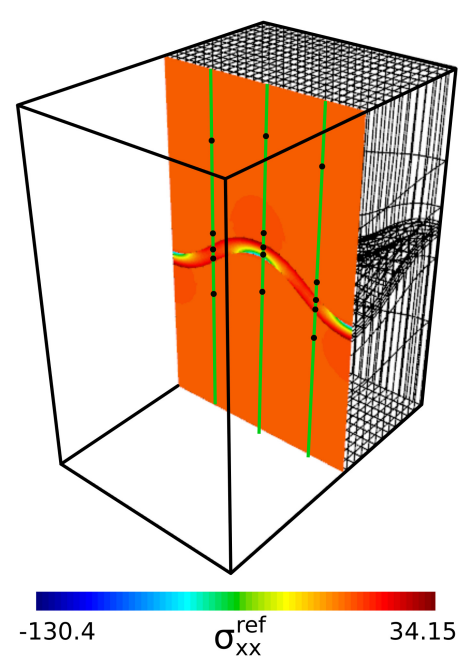

(d)

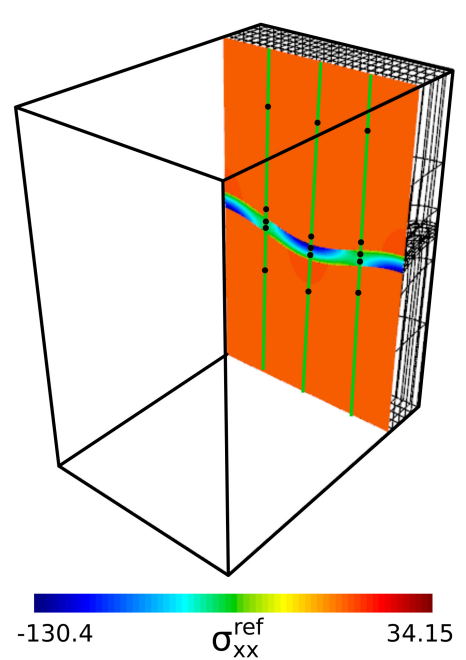

(e)

Figure 2 a) Initial configuration of the model. b) Deformed model at $t_{f}=50$. Top and bottom layer horizons are only shown in these figures in order to see the fold. c), d) and e) show different slices where the stress $\sigma_{x x}^{\text {ref }}$ (in the $x$ direction) is computed using PINK-3D. Virtual wells are represented as green lines and $\sigma_{x x}$ and $\sigma_{y y}$ are extracted on the black dots.

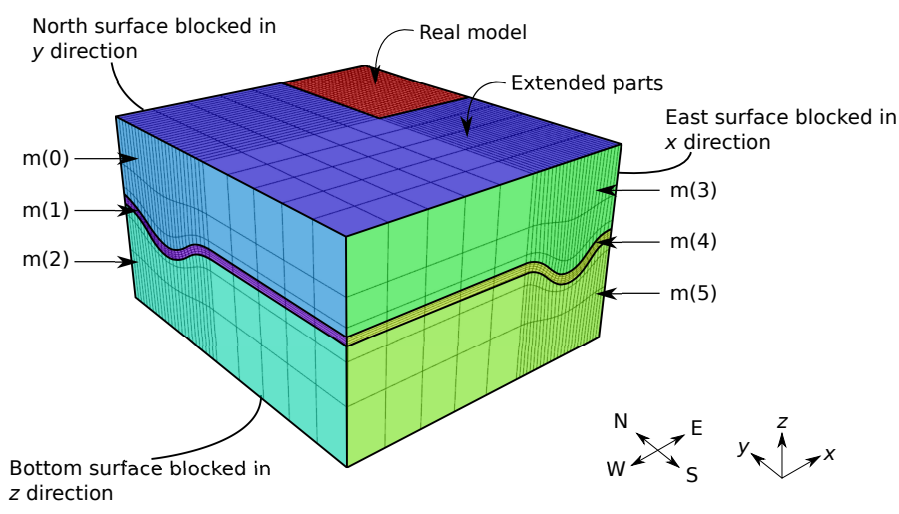

Figure 3 Model extensions in South and West directions and the Neumann conditions location.

\subsection{Results and comparison between the reference and the computed stress}

The CMA-ES results are shown in Fig. 4 and Fig. 5. CMAES succeeds to converge with a final misfit function value of $S\left(\boldsymbol{m}_{\text {final }}\right)=1.34$ in 301 iterations or 3612 evaluations of the misfit function. It manages to find the most suitable Neumann boundary conditions applied on the model that minimize the misfit between the reference stress $\sigma_{x x}^{\text {ref }}$ and the computed stress $\sigma_{x x}^{\text {computed }}$. The evolution of the mean of each model parameter is shown in Fig. 5. The reader should be aware that a negative model parameter matches with extensive boundary conditions and positive values match with compressive boundary conditions. Fig. 5 shows that the retrieved boundary conditions are far from being homogeneous with a high contrast between the ones that are applied on the strong layer and the ones applied on the matrix. Stress computation using linear elastic model is ordinary in geomechanics. Neumann conditions used are often set to a constant value [Lovely et al., 2012] or to a single linear function [Gunzburger and Magnenet, 2014] in order to run forward simulations. This result demonstrates that these classical modeling strategies could lead to high errors on the in-situ stress estimation.

As shown in Fig. $6 \mathrm{a}$ and $\mathrm{b}$, the $\sigma_{x x}$ distribution is different between the layer and the matrix. To compare the retrieved stress $\sigma_{x x}^{\text {computed }}$ with the reference stress $\sigma_{x x}^{\text {ref }}$, we use two different color scales. One is used to compare the stress in the layer (results are presented in Fig. 7b for the middle slice) and the other is used to compare the stress in the matrix (results are presented in Fig. 7c for the middle slice). A global error is computed using a difference which is normalized by the standard deviation for each distribution (Eq. 8). The error is displayed on Fig. 7d. We have developed an online tool to visualize the stress fields and error in 


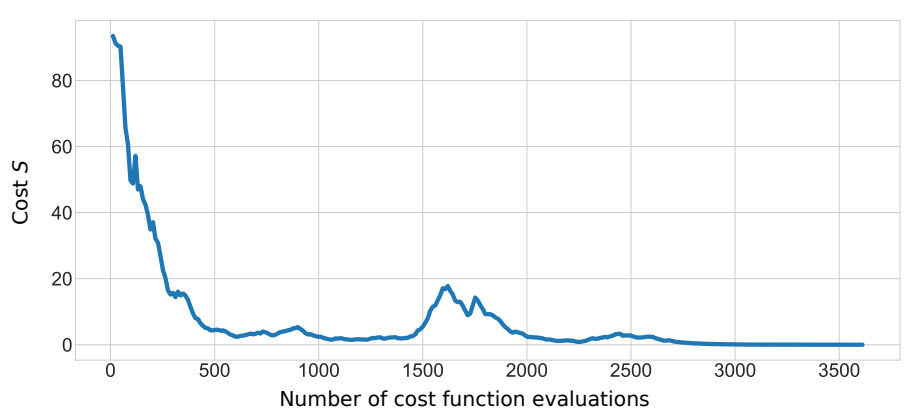

Figure 4 Minimum misfit function values for each generation of the CMA-ES algorithm

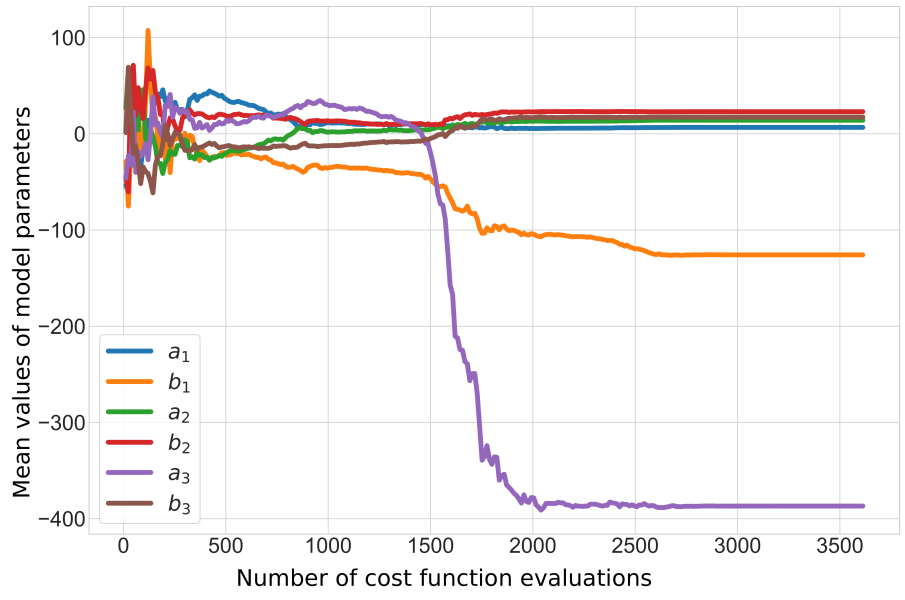

Figure 5 Mean values of model parameters for each generation of the CMA-ES algorithm

$m(0)$ : top East Neumann condition mean value ; $m(1)$ : mid West Neumann condition mean value ; $m(2)$ : bottom West Neumann condition mean value

$m(3)$ : top South Neumann condition mean value ; $m(4)$ : mid South Neumann condition mean value ; $m(5)$ : bottom South Neumann condition mean value.

the whole model using an interactive slicer accessible here: http://mazuyer-cageo2017.ring-team.org.

$$
\text { error }=\frac{\left\|\sigma_{x x}^{\text {computed }}-\sigma_{x x}^{\text {ref }}\right\|}{\text { std }}
$$

Fig. 7b shows that $\sigma_{x x}^{\text {computed }}$ presents similar variations to $\sigma_{x x}^{\text {ref }}$ in the layer. Indeed, the inverse method manages to retrieve the compressive stress area nearby the fold lower horizon and the extensive stress area nearby the fold upper horizon. It means that the solving of Eq. 2 using the finite element method can reproduce the stress distribution inside an isopach fold, even if it was generated with a large strain simulation. However, the inverse method failed to retrieve the most compressive and extensive areas. This observation can also be noted by looking at the histograms of Fig. $6 \mathrm{~b}$ and d: the $\sigma_{x x}^{\text {ref }}$ range is $[-130,34]$ whereas $\sigma_{x x}^{\text {computed }}$ range is $[-107,12]$.

Fig. $7 \mathrm{c}$ shows that $\sigma_{x x}^{\text {computed }}$ in the matrix is more subject to side effects, even if the boundaries were extended, particularly nearby the bottom of the model. $\sigma_{x x}^{\text {ref }}$ variation is sharper than $\sigma_{x x}^{\text {computed }}$. Indeed, the histograms of Fig. $6 \mathrm{a}$ and c show that the $\sigma_{x x}^{\text {computed }}$ distribution is closer to a perfect gaussian distribution than the $\sigma_{x x}^{\text {ref }}$ one.

The error which is displayed in Fig. 7d shows that the high errors are mainly concentrated in the matrix, nearby the fold lower and upper surfaces. The error remains globally low in

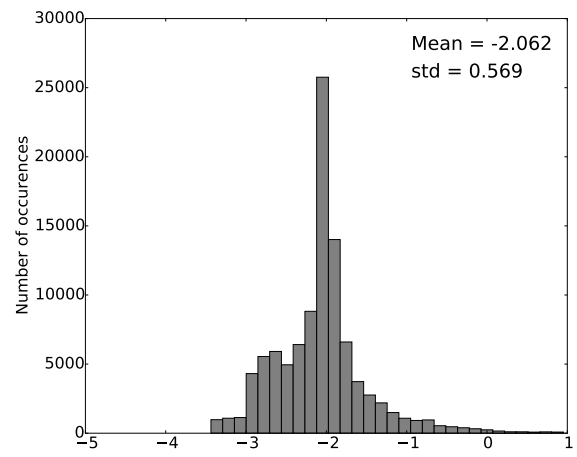

(a) $\sigma_{x x}^{\text {ref }}$ distribution in the matrix

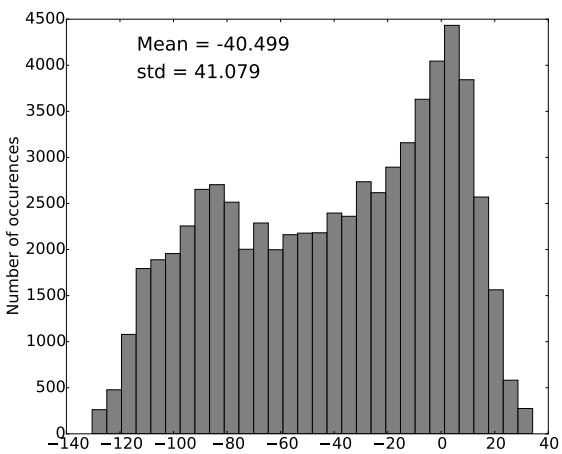

(b) $\sigma_{x x}^{\text {ref }}$ distribution in the layer

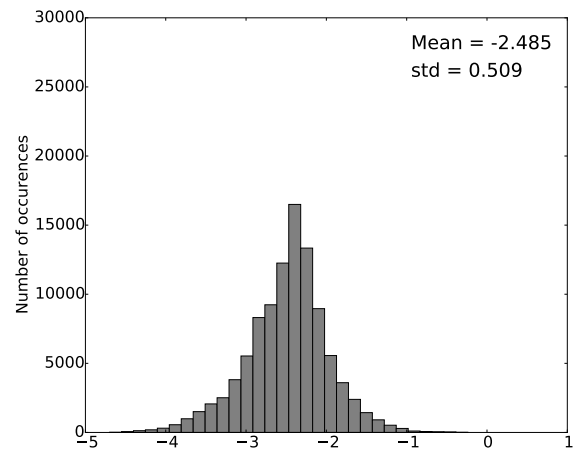

(c) $\sigma_{x x}^{\text {computed }}$ distribution in the matrix

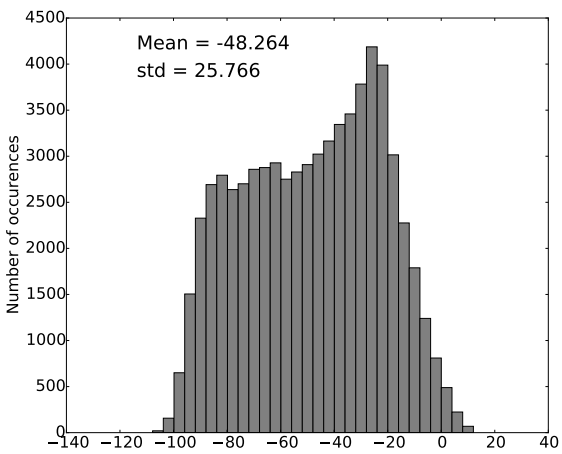

(d) $\sigma_{x x}^{\text {computed }}$ distribution in the layer

Figure 6 Distributions of the computed and reference $\sigma_{x x}$ in the matrix and the layer. In both cases, $\sigma_{x x}$ is sampled using the 27 interpolation nodes of each mesh element 
the area of the data points. The differences between $\sigma_{x x}^{\text {ref }}$ and $\sigma_{x x}^{\text {computed }}$ can be a consequence of a wrong choice of elastic parameters. Indeed, the ratio between $E_{L}$ and $E_{M}$ may not be the same as the ratio between $\eta_{L}$ and $\eta_{M}$ used for the largestrain simulation and the Poisson's ratio was arbitrarily fixed. Moreover, the linear elastic assumption may not be sufficient to well reproduce the stress state in the area where we expect to exceed yield criterion such as nearby the fold lower and upper surfaces.

\section{Conclusions ANd PERSPECtives}

We have presented a complete method to estimate the virgin stress in the reservoirs and the overburden with the integration of different libraries. Even if the work was presented with the finite element method to solve the forward problem and the CMA-ES algorithm to optimize the misfit function, a particular effort was made to allow the developer the possibility to implement his own simulator or optimization algorithm, or to plug-in an existing library like we did with MFEM. This software integration gives to the user or the developer a complete control of the methods used. The dependency with the RINGMesh library provides data structures that can handle 3D meshes of complex geological structures. Using 3D meshes while resolving the boundary value problem presented in section 2 is essential to take into account the effects of the geolog ical structures on the stress. Faults are not taken into account in the presented work as it is only based on linear elasticity. Future study will focus on the integration of fault behaviour in the forward problem. To keep an elastic behaviour which guarantees low computational cost, one method could be to modify the elastic parameters nearby the faults [Faulkner et al., 2006].

Even if the method was tested on a simple case on which we had the control on the data points, real case studies often provide a poor number of stress data so the inverse problem becomes ill-conditioned. The CMA-ES algorithm can take into account a large amount of model parameters and it has shown good performances to solve ill-posed problem. The CMA-ES output gives a set of heterogeneous Neumann conditions that are not the real boundary conditions in place and that would not have been found without the inverse method. The application of these boundary conditions shows that the retrieved stress presents very similar variations as the reference one. To improve the precision of the algorithm, we are working on a way to preconstrain the model with an initial stress. This initial stress could be the result of the rock weight and the stress distribution that we can deduce by propagating the stress from a data point, inside a fold or nearby the faults where we know the stress patterns. This rough stress estimation could improve the computation time and produce more precise results.

In this study, the input data used for the inversion was supposed to be free of uncertainties; this is unrealistic since data acquisitions are always subject to measurement errors. A practical way for dealing with noisy data in the CMA-ES algorithm is to increase the termination threshold according to data uncertainty level. Indeed, the diagonal elements of the posterior covariance will be larger and the individuals of the final generation more scattered. Finally, the posterior mean and covariance matrix could be used to draw sets of Neumann conditions corresponding to sets of 3D stress fields accounting for the uncertainties on the wellbore data.

\section{ACKNOWLEDGEMENT}

This work was performed in the frame of the RING project at Université de Lorraine. We would like to thank the industrial and academic sponsors of the Gocad Research Consortium managed by ASGA for their support, in particular Total for additional support for Antoine Mazuyer Ph.D. We are also very grateful to Guillaume Caumon who has initiated the project. We thank very much the reviewers of our paper for the attention they paid to our work and their comments that helped us to improve the quality of the manuscript.

\section{A USE OF CMA-ES TO OPTIMIZE THE MISFIT FUNCTION}

CMA-ES is based on a population that evolves until a termination criterion is reached. A simplified flowchart of the algorithm is given in Fig. 8. A population is described by a vector $\boldsymbol{m}$ which has the size of the number of parameters to optimize (i.e. the number of parameters to invert). The initial population of model $m^{(0)}$ is generated from an initial mean $\bar{m}^{(0)}$ and an initial covariance matrix $C^{(0)}$ (not to be confused with the elasticity matrix) given by the user. The size of the population is denoted by $\lambda$. At a given iteration $g+1$, an individual $\boldsymbol{m}_{k}^{(g+1)}(k \in \llbracket 1, \lambda \rrbracket)$ of the new population is created from the mean and the covariance matrix of the previous iteration (Fig. 8a):

$$
\boldsymbol{m}_{k}^{(g+1)}=\overline{\boldsymbol{m}}^{(g)}+\sigma^{(g)} \mathscr{N}\left(0, \boldsymbol{C}^{(g)}\right)
$$

$\sigma^{(g)}$ is the step-size. It controls the "overall scale" of the distribution and $\mathscr{N}$ denotes the gaussian distribution. To build a new population from this state, CMA-ES uses a two-step algorithm: the update of the mean and the covariance matrix adaptation. For one iteration, CMA-ES evaluates the misfit function defined in Eq. 7. In our case, it means that the boundary-problem defined in Eq. 2 has to be solved $\lambda$ times for one iteration. To compute the mean at iteration $g+1$, CMA-ES uses the weighted sum over the $\mu$ best individuals (i.e. the model parameters that minimize the misfit function, Fig. $8 \mathrm{~b}$ ).

$$
\overline{\boldsymbol{m}}^{(g+1)}=\sum_{i=1}^{\mu} w_{i} \boldsymbol{m}_{i: \lambda}^{(g+1)}
$$

where $w_{i}$ are the weight coefficients, and $\boldsymbol{m}_{i: \lambda}^{(g+1)}$ the $i$-th best individual at iteration $g+1\left(S\left(\boldsymbol{m}_{1: \lambda}^{(g+1)}\right) \leq S\left(\boldsymbol{m}_{2: \lambda}^{(g+1)}\right) \leq \ldots \leq\right.$ $\left.S\left(\boldsymbol{m}_{\lambda: \lambda}^{(g+1)}\right)\right)$.

Two methods are combined to update the covariance matrix $C$ : the first one is the rank- $\mu$ update [Hansen et al., 2003] and the second one is the rank-one update.

The rank- $\mu$ update involves the distance between the mean at the previous iteration and the $\mu$ best individuals (Fig. 8c):

$$
\boldsymbol{C}^{(g+1)}=\left(1-c_{\mu}\right) \boldsymbol{C}^{(g)}+c_{\mu} \underbrace{\sum_{i=1}^{\mu} w_{i} \mathbf{y}_{i: \lambda}^{(g+1)}\left(y_{i: \lambda}^{(g+1)}\right)^{T}}_{\begin{array}{c}
\text { Contribution of the } \\
\text { rank } \mu \text { update }
\end{array}}
$$

with $c_{\mu}$ the "learning rate" which weights the rank- $\mu$ update and $\boldsymbol{y}_{i: \lambda}^{(g+1)}=\frac{\boldsymbol{m}_{i: \lambda}^{(g+1)}-\overline{\boldsymbol{m}}^{(g)}}{\sigma^{(g)}}$.

The rank-one update contribution involves the distance between the mean at the previous iteration and the current mean. 


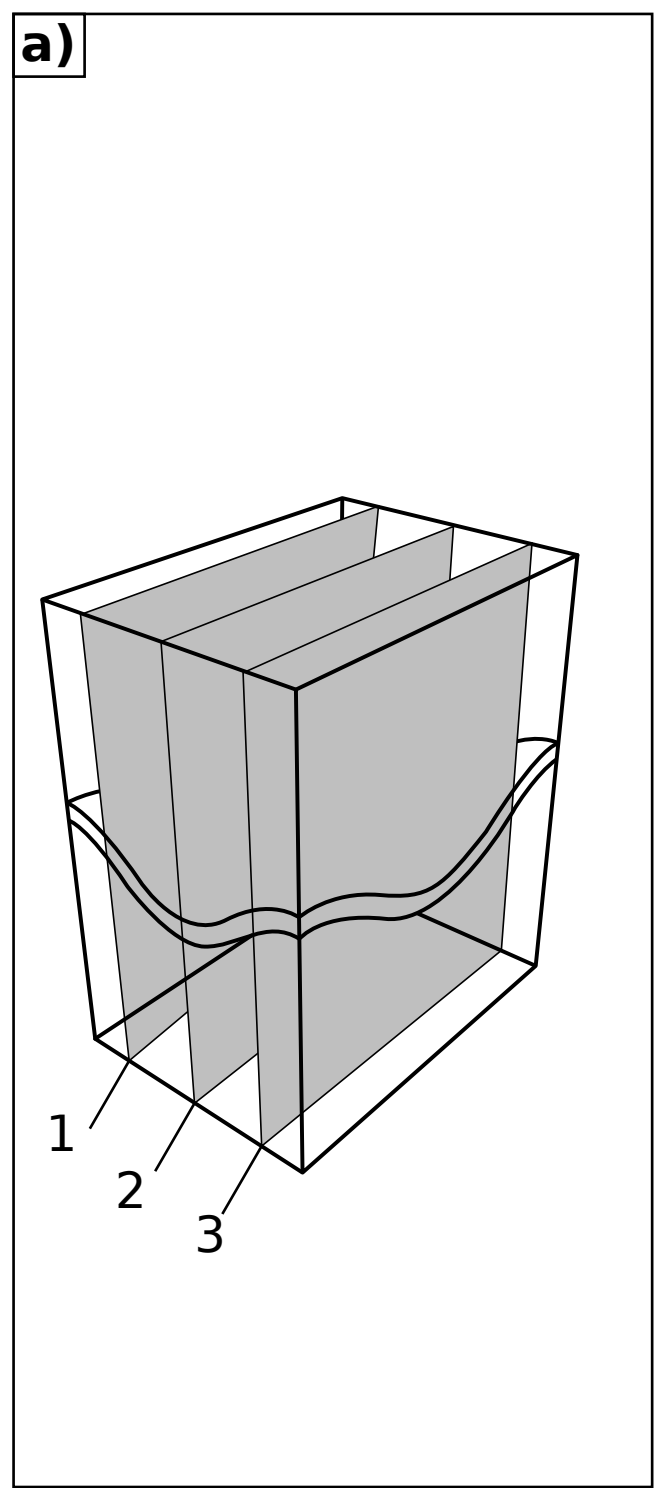

b)

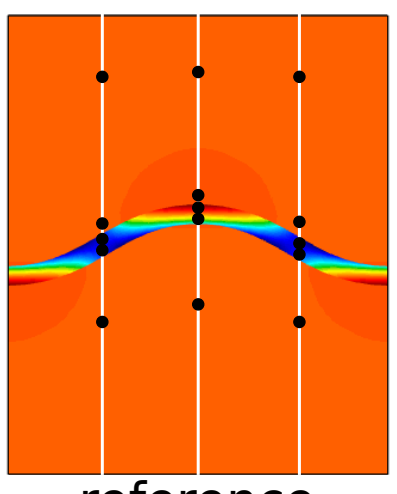

reference
Slice 2)

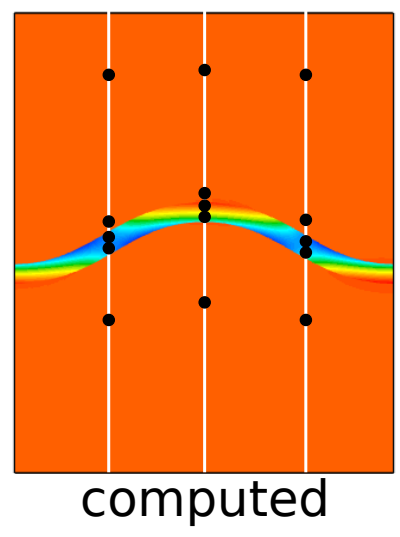

$\sigma_{x x}$

34.15 c)

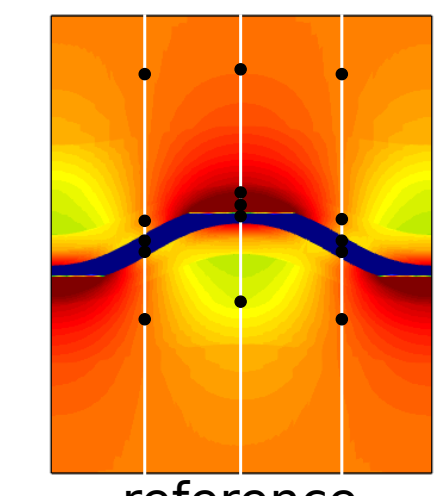

reference
Slice 2)

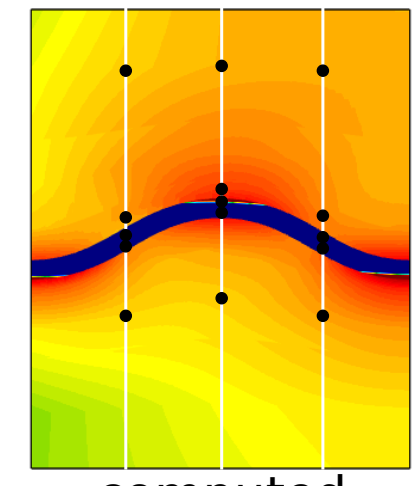

computed

d)

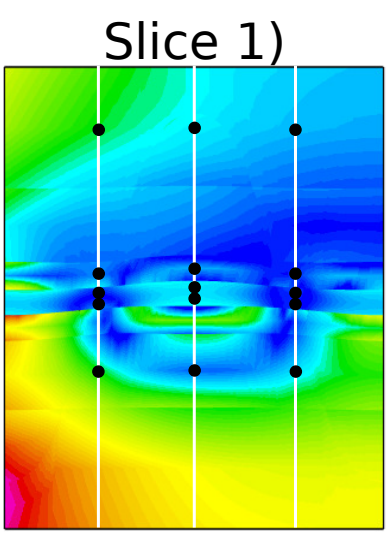

Slice 2)

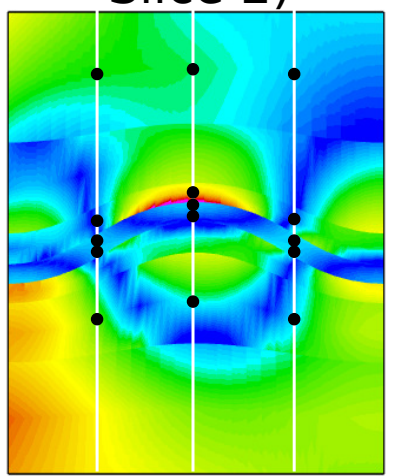

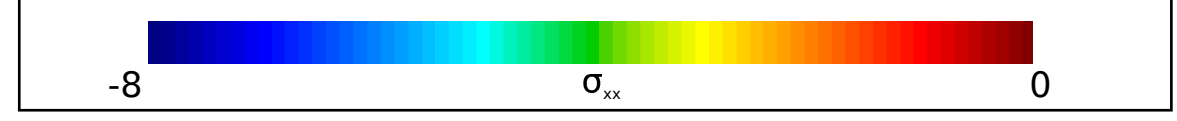


a)

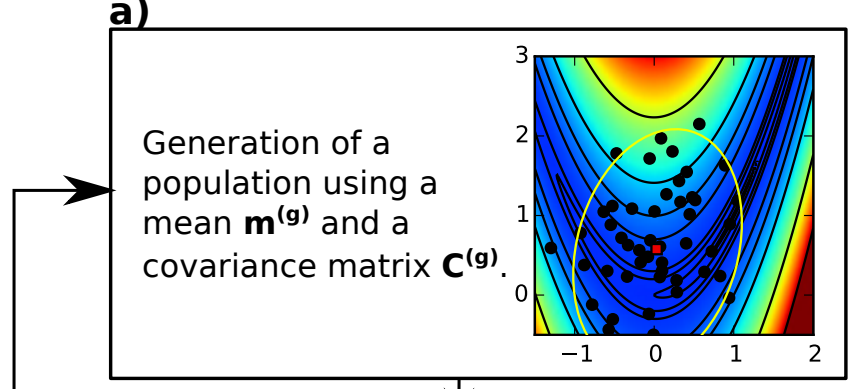

b)

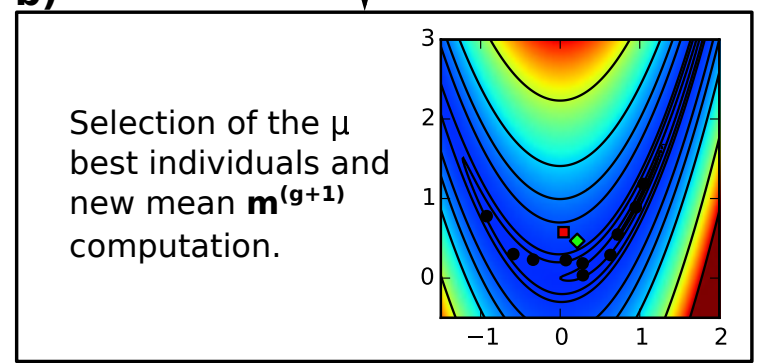

c)

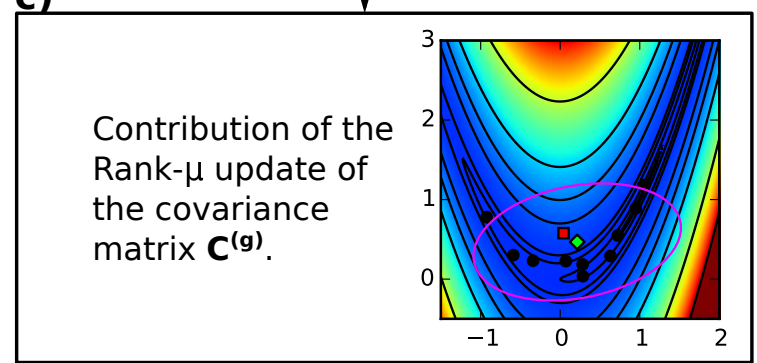

d)

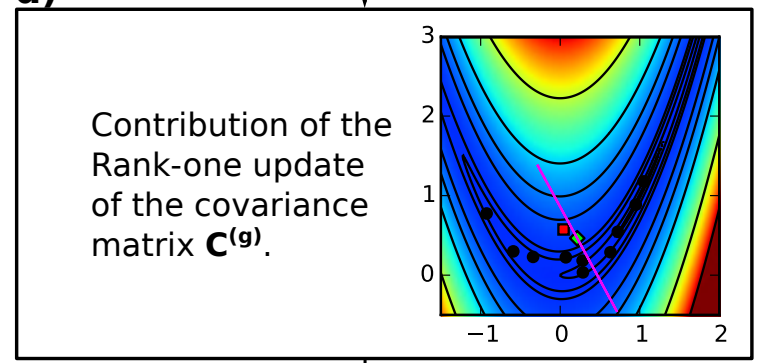

e)

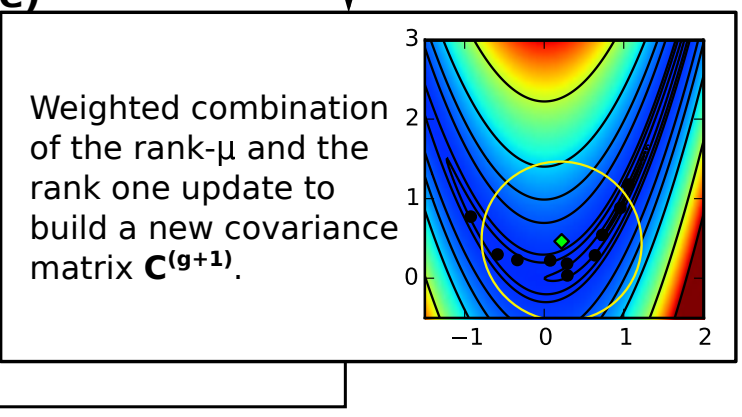

Figure 8 Flowchart of the CMA-ES algorithm illustrated with the Rosenbrock function in a 2D space optimised using a population of 50 individuals. Each black dot is an individual of the population. Red dot is the mean at the generation $(g)$ while the green dot is the mean at the generation $(g+1)$. The yellow ellipses are the graphical representations of the covariance matrices. The pink ellipses are the graphical representations of the contributions to the update of the covariance matrix.
It can be assimilated to steepest descent in classical gradientbased methods (Fig. 8d)

$$
\boldsymbol{C}^{(g+1)}=\left(1-c_{1}\right) \boldsymbol{C}^{(g)}+c_{1} \underbrace{\boldsymbol{p}_{\boldsymbol{c}}^{(g+1)}\left(\boldsymbol{p}_{\boldsymbol{c}}^{(g+1)}\right)^{T}}_{\begin{array}{c}
\text { Contribution of the } \\
\text { rank-one update }
\end{array}}
$$

with $c_{1}$ the learning rate which weights the rank-one update, and $\boldsymbol{p}_{\boldsymbol{c}}{ }^{(g)}$ the cumulation path such that:

$$
\boldsymbol{p}_{\boldsymbol{c}}^{(g+1)}=\left(1-c_{c}\right) \boldsymbol{p}_{\boldsymbol{c}}^{(g)}+\sqrt{c_{c}\left(2-c_{c}\right) \mu_{\mathrm{eff}}} \frac{\overline{\boldsymbol{m}}^{(g+1)}-\overline{\boldsymbol{m}}^{(g)}}{\sigma^{(g)}}
$$

with $c_{c}$ the learning rate for the cumulation and $\mu_{\text {eff }}$ the variance effective selection mass computed from the weight coefficients $\mu_{\mathrm{eff}}=\left(\sum_{i=1}^{\mu} w_{i}^{2}\right)^{2}$.

Finally, the rank- $\mu$ update and the rank-one update are combined to compute $\boldsymbol{C}^{(g+1)}$ (Fig. 8e):

$$
\begin{aligned}
\boldsymbol{C}^{(g+1)}=\left(1-c_{1}-c_{\mu}\right) \boldsymbol{C}^{(g)} & +\underbrace{c_{1} \boldsymbol{p}_{\boldsymbol{c}}^{(g+1)}\left(\boldsymbol{p}_{\boldsymbol{c}}{ }^{(g+1)}\right)^{T}}_{\text {rank-one update }} \\
& +\underbrace{c_{\mu} \sum_{i=1}^{\mu} w_{i} \boldsymbol{y}_{i: \lambda}^{(g+1)}\left(\boldsymbol{y}_{i: \lambda}^{(g+1)}\right)^{T}}_{\text {rank }-\mu \text { update }}
\end{aligned}
$$

Learning rates $c_{c}, c_{\mu}$ and $c_{1}$ can be set by the user weight both rank-one and rank- $\mu$ update or estimated by the algorithm ${ }^{1}$.

\section{REFERENCES}

M. Addis. The stress-depletion response of reservoirs. In SPE Annual Technical Conference and Exhibition. Society of Petroleum Engineers, 1997. ISBN 978-1-55563-399-8. doi: 10.2118/38720-MS. (Cited page 1)

B. Amadei and O. Stephansson. Rock Stress and Its Measurement. Springer Netherlands, Dordrecht, 1997. ISBN 978-94-010-6247-3. doi: 10.1007/ 978-94-011-5346-1. (Cited pages 1 and 4)

A. Auger, N. Hansen, J. M. Perez Zerpa, R. Ros, and M. Schoenauer. Experimental comparisons of derivative free optimization algorithms (invited talk). In Lecture Notes in Computer Science (including subseries Lecture Notes in Artificial Intelligence and Lecture Notes in Bioinformatics), volume 5526 LNCS, pages 3-15. Springer Berlin Heidelberg, 2009. ISBN 3642020100. doi: 10.1007/978-3-642-02011-7_3. (Cited page 4)

T. Bäck and H.-P. Schwefel. An overview of evolutionary algorithms for parameter optimization. Evolutionary computation, 1(1):1-23, 1993. (Cited page 4)

K. Beaty, D. Schmitt, and M. Sacchi. Simulated annealing inversion of multimode rayleigh wave dispersion curves for geological structure. Geophysical Journal International, 151(2):622-631, 2002. (Cited page 4)

M. Bosch, T. Mukerji, and E. F. Gonzalez. Seismic inversion for reservoir properties combining statistical rock physics and geostatistics: A review. Geophysics, 75(5):165-176, 2010. ISSN 0016-8033. doi: 10.1190/1. 3478209. (Cited page 2)

A. Botella. Génération de maillages non structurés volumiques de modèles géologiques pour la simulation de phénomènes physiques. $\mathrm{PhD}$ thesis, Université de Lorraine, 2016. (Cited page 4)

A. Botella, B. Lévy, and G. Caumon. Indirect unstructured hex-dominant mesh generation using tetrahedra recombination. Computational Geosciences, 20 (3):437-451, 2016. ISSN 1420-0597. doi: 10.1007/s10596-015-9484-9. (Cited page 4)

J. Clark. A hydraulic process for increasing the productivity of wells. Journal of Petroleum Technology, 1(01):1-8, 1949. ISSN 0149-2136. doi: 10.2118/ 949001-G. (Cited pages 1 and 4)

R. Das. A simulated annealing-based inverse computational fluid dynamics model for unknown parameter estimation in fluid flow problem. International Journal of Computational Fluid Dynamics, 26(9-10):499-513, 2012. (Cited page 4)

${ }^{1}$ Practical information about the algorithm is detailed on the main page of CMA-ES: https://www. lri.fr/ hansen/cmaesintro.html 
R. Das. Estimation of feasible materials and thermal conditions in a trapezoidal fin using genetic algorithm. Proceedings of the Institution of Mechanical Engineers, Part G: Journal of Aerospace Engineering, 230(13):2356-2368, 2016. (Cited page 4)

R. Das and D. K. Prasad. Prediction of porosity and thermal diffusivity in a porous fin using differential evolution algorithm. Swarm and Evolutionary Computation, 23:27-39, 2015. (Cited page 4)

C. V. Deutsch and P. W. Cockerham. Practical considerations in the application of simulated annealing to stochastic simulation. Mathematical Geology, 26 (1):67-82, 1994. (Cited page 4)

D. R. Faulkner, T. M. Mitchell, D. Healy, and M. J. Heap. Slip on 'weak' faults by the rotation of regional stress in the fracture damage zone. Nature, 444(7121):922-925, 2006. ISSN 0028-0836. doi: 10.1038/nature05353. (Cited pages 1 and 8)

K. Gallagher and M. Sambridge. Genetic algorithms: a powerful tool for largescale nonlinear optimization problems. Computers \& Geosciences, 20(7-8): 1229-1236, 1994. (Cited page 4)

T. V. Gerya and D. A. Yuen. Robust characteristics method for modelling multiphase visco-elasto-plastic thermo-mechanical problems. Physics of the Earth and Planetary Interiors, 163(1):83-105, 2007. ISSN 00319201. doi: 10.1016/j.pepi.2007.04.015. (Cited page 1)

R. Giot. Interprétation des mesures de contraintes par relaxation dans les formations argileuses profondes. PhD thesis, Vandoeuvre-les-Nancy, INPL, 2004. (Cited page 4)

Y. Gunzburger and V. Magnenet. Stress inversion and basement-cover stress transmission across weak layers in the Paris basin, France. Tectonophysics, 617:44-57, 2014. doi: 10.1016/j.tecto.2014.01.016. (Cited page 6)

N. Hansen. CMA-ES: CMA-ES written in ANSI C in a fairly object-oriented style. 2016. URL https://github.com/cma-es/c-cmaes. (Cited page 4)

N. Hansen and A. Ostermeier. Completely derandomized self-adaptation in evolution strategies. Evolutionary computation, 9(2):159-95, 2001. ISSN 1063-6560. doi: 10.1162/106365601750190398. (Cited page 4)

N. Hansen, S. D. Müller, and P. Koumoutsakos. Reducing the Time Complexity of the Derandomized Evolution Strategy with Covariance Matrix Adaptation (CMA-ES). Evolutionary Computation, 11(1):1-18, 2003. ISSN 1063-6560. doi: 10.1162/106365603321828970. (Cited page 8)

R. Hassani, D. Jongmans, and J. Chéry. Study of plate deformation and stress in subduction processes using two-dimensional numerical models. Journal of Geophysical Research: Solid Earth, 102(B8):17951-17965, 1997. ISSN 01480227. doi: 10.1029/97JB01354. (Cited page 1)

M. K. Hubbert and D. G. Willis. Mechanics Of Hydraulic Fracturing. Petroleum Transactions, 210:153-168, 1957. (Cited page 4)

S. Kirkpatrick, C. D. Gelatt, M. P. Vecchi, et al. Optimization by simulated annealing. science, 220(4598):671-680, 1983. (Cited page 4)

P. Lovely, E. Flodin, C. Guzofski, F. Maerten, and D. D. Pollard. Pitfalls among the promises of mechanics-based restoration: Addressing implications of unphysical boundary conditions. Journal of Structural Geology, 41:47-63, 2012. doi: 10.1016/j.jsg.2012.02.020. (Cited page 6)

A. Madyarov and A. Savitski. Numerical algorithm for constructing 3D initial stress field matching field measurements. 44th U.S. Rock Mechanics Symposium and 5th U.S.-Canada Rock Mechanics Symposium, 2010. (Cited pages 1 and 4)

L. Maerten, F. Maerten, M. Lejri, and P. Gillespie. Geomechanical paleostress inversion using fracture data. Journal of Structural Geology, 89:197-213, 2016. (Cited page 1)

MFEM. MFEM: Modular finite element methods. 2017. URL mfem.org. (Cited page 4)

Paradigm. SKUA-GOCAD. 2017. URL http://www.pdgm.com/ products/skua-gocad/. (Cited page 4)

J. Pellerin, A. Botella, F. Bonneau, A. Mazuyer, B. Chauvin, B. Lévy, and G. Caumon. RINGMesh: A programming library for developing mesh-based geomodeling applications. Computers \& Geosciences, 2017. (Cited page 4)

L. M. Rios and N. V. Sahinidis. Derivative-free optimization: a review of algorithms and comparison of software implementations. Journal of Global Optimization, 56(3):1247-1293, 2013. ISSN 0925-5001. doi: 10.1007/ s10898-012-9951-y. (Cited page 2)

J. Salençon. Handbook of continuum mechanics: general concepts thermoelasticity. Springer Science \& Business Media, 2012. (Cited page 2)

A. Tarantola. Inverse Problem Theory and Methods for Model Parameter Estimation. Society for Industrial and Applied Mathematics, 2005. ISBN 978-0 89871-572-9. doi: 10.1137/1.9780898717921. (Cited pages 1, 2, and 4)

M. von Tscharner and S. M. Schmalholz. A 3-D Lagrangian finite element algorithm with remeshing for simulating large-strain hydrodynamic instabilities in power law viscoelastic fluids. Geochemistry, Geophysics, Geosystems,
16(1):215-245, 2015. ISSN 15252027. doi: 10.1002/2014GC005628. (Cited pages 2 and 5)

D. Wiprut and M. D. Zoback. Fault reactivation, leakage potential, and hydrocarbon column heights in the northern north sea. Norwegian Petroleum Society Special Publications, 11(C):203-219, 2002. ISSN 09288937. doi: 10.1016/S0928-8937(02)80016-9. (Cited page 1)

O. C. Zienkiewicz and R. L. Taylor. The finite element method: solid mechanics, volume 2. Butterworth-heinemann, 2000. (Cited page 2)

M. Zoback, M. Zoback, V. S. Mount, J. Suppe, J. P. Eaton, J. H. Healy, D. Oppenheimer, P. Reasenberg, L. Jones, C. B. Raleigh, I. G. Wong, O. Scotti, and C. Wentworth. New evidence on the state of stress of the San Andreas Fault system, 1987. ISSN 10959203. (Cited page 1)

M. D. Zoback. Reservoir Geomechanics. Cambridge University Press, 2007. ISBN 1107320089. (Cited pages 1 and 4)

M. D. Zoback, C. a. Barton, M. Brudy, D. a. Castillo, T. Finkbeiner, B. R. Grollimund, D. B. Moos, P. Peska, C. D. Ward, and D. J. Wiprut. Determination of stress orientation and magnitude in deep wells. International Journal of Rock Mechanics and Mining Sciences, 40(7-8):1049-1076, 2003. ISSN 13651609. doi: 10.1016/j.ijrmms.2003.07.001. (Cited page 1) 